\title{
Flt3 ligand expanded bone marrow mesenchymal stem cells for cartilage tissue engineering
}

\author{
Chang M Guo ${ }^{1}$, Kelly YW Chan ${ }^{2}$, Philip Cheang ${ }^{2}$, Kam M Hui ${ }^{3}$, Ivy AW Ho ${ }^{3}$, Paula YP Lam ${ }^{3}$ \\ ${ }^{1}$ Department of Orthopedic, Singapore General Hospital, Singapore 169608; ${ }^{2}$ Division of BioEngineering, School of Chemical and \\ Biomedical Engineering, Nanyang Technological University, Singapore 639798; ${ }^{3}$ Gene Vector Laboratory, Division of Cellular \\ and Molecular Research, National Cancer Centre, Singapore 169610
}

The aim of the present in vitro study is to investigate the expansion effects of Flt3 ligand (FIt3L) as an individual stimulant for human mesenchymal stem cells (hMSCs), as well as in combination with other co-stimulants. Normal medium is served as a control, whereas FIt3 $\mathrm{L}$ in combination with other growth factors was used to distinguish the difference between Flt3L-mediated effects and growth factor induced effects on hMSCs. Flt3L alone is known to generate large numbers of dendritic cells from hematopoietic progenitor and stem cells and to augment immune responses in vivo. Flt3L can also be used to stimulate and expand myeloid, lymphoid progenitor cells, dendritic and natural killer cells ex vivo.

Our results showed that intracellular Flt3 receptors have been found to exist in hMSCs and expansion of hMSCs is highly dependant on the time, duration and concentration of FIt3 $\mathrm{L}$ as well as in combination with other growth factors, which work together in a synergistic effect to influence the expansion of hMSCs. Expansion of hMSCs using FIt3L and other growth factors does not affect the cell's pluripotency, as shown by characterisation of hMSCs before and after expansion.

We are the first study to comprehensively describe the effects of Fit3L on hMSCs and our results are of special clinically interest regarding the stimulation of bone healing in orthopaedic and traumatic surgery.

Keywords: mesenchymal stem cells, Flt3 ligand, expansion

Cell Research (2008) 18:s56. doi: 10.1038/cr.2008.146; published online 4 August 2008

Correspondence: Changming Guo

E-mail: googcm@sgh.com.sg 